\title{
REVIEW
}

\section{Molecular histology in the study of solid tumours}

\author{
R J Campbell, M Pignatelli
}

Dominant oncogenes and tumour suppressor gene abnormalities are crucial events in human cancer. Many molecular techniques are used to identify these abnormalities, including single strand conformational polymorphism, the polymerase chain reaction, cloning, and sequencing, although the biological relevance of these changes is not always apparent. Immunohistochemistry $(\mathrm{ICH})$ or western blotting of abnormal gene products can provide information about their cellular localisation and expression in neoplastic versus normal cells, and can sometimes give a clue to their function. For example, ICH has shown how loss of the intercellular adhesion molecule E-cadherin, or abnormal localisation from the cell membrane to the cytoplasm, correlates with a diffuse tumour phenotype and a less favourable clinical outcome. Similarly, ICH of $\beta$-catenin (a protein that binds E-cadherin and is essential for its function) has shown abnormal cellular localisation in the nucleus in a variety of human malignancies; in particular, colorectal carcinomas, where abnormal forms of the adenomatous polyposis coli gene product cause nuclear and cytoplasmic sequestration of $\beta$-catenin. Such studies show how morphological assessment can sometimes provide insight into molecular function and dysfunction in human malignancy.

See end of article for authors' affiliations

........................

Correspondence to: Professor Pignatelli,

Division of Histopathology, Department of Pathology and Microbiology,

University of Bristol, Bristol Royal Infirmary,

Marlborough Street, Bristol

BS2 8HW, UK;

Massimo.Pignatelli@

bristol.ac.uk

Accepted for publication 15 March 2001

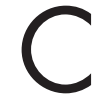

ancer is ultimately a genetic disease and as such the focus for much cancer research has been directed towards the understanding of how many oncogenes and tumour suppressor genes become dysfunctional in human malignancy. Recently, the range of methods to examine these abnormalities has widened and many new and powerful molecular techniques have become available. These include the detection of mutations using the polymerase chain reaction and DNA sequencing, and comparative genomic hybridisation on genomic microarrays to detect gene amplifications and deletions on a genome wide basis. However, these new techniques have yet to find a role in the routine work of the diagnostic histopathology laboratory for several reasons. They can be expensive to set up or run, or produce poor results from routine formalin fixed paraffin wax embedded specimens and thus require specimens to be processed differently. In addition, although these techniques will often very accurately determine the presence or absence of mutations, determining their precise
J Clin Pathol: Mol Pathol 2002;55:80-82

biological importance is not always easy from such data.

However, there are a range of complementary techniques that have already found a place in the work of many histopathology laboratories, either as part of diagnostic or research work. These procedures allow the direct visualisation of abnormal gene products and include immunohistochemistry, western blotting, and in situ hybridisation to evaluate mRNA expression. Immunohistochemistry in particular is extensively used as a diagnostic tool to determine the presence or absence of particular proteins in routinely fixed and embedded samples. It is inexpensive, relatively easy to perform, and can help visualise cell types in vivo that may harbour abnormalities. In general, techniques such as these can (1) provide useful information regarding the location of abnormal gene products (either between different cell types or within subcellular compartments); (2) provide information on the level of gene expression of such products in tumour cells compared with normal cells; and (3) in certain instances, allow insights into the function of particular altered genes and their products. This approach neatly links the molecular biology of any cancer under scrutiny with its histological characteristics and behaviour and can be given the epithet "molecular histology", a term first coined by Edelman and Crossin in 1991, ${ }^{1}$ and further revisited by Smith and Pignatelli in 1997..$^{2}$ Molecular histology is therefore an explanation of the morphological characteristics of a tissue in terms of the molecules present and the functional interactions between them.

The study of the role of the intercellular adhesion molecule E-cadherin in malignancy is a particular area where a "molecular histological" approach has been fruitful, and the rest of this discussion will focus on the important work in this area, and how molecular aspects have been shown to relate to the observed morphology, and vice versa.

\section{THE CADHERIN-CATENIN COMPLEX}

Cadherins are a family of calcium dependant cell adhesion molecules with an important role in normal cell adhesion. They exist as cell surface, membrane spanning glycoproteins with molecular weights around $120 \mathrm{kDa}$. Different cadherins are found on different tissues; thus, E-cadherin is found in all normal epithelia, P-cadherin binds trophoblastic cells together and is present in

Abbreviations: APC, adenomatous polyposis coli; $\mathrm{CIN}$, cervical intraepithelial neoplasia; EGFR, epidermal growth factor receptor 


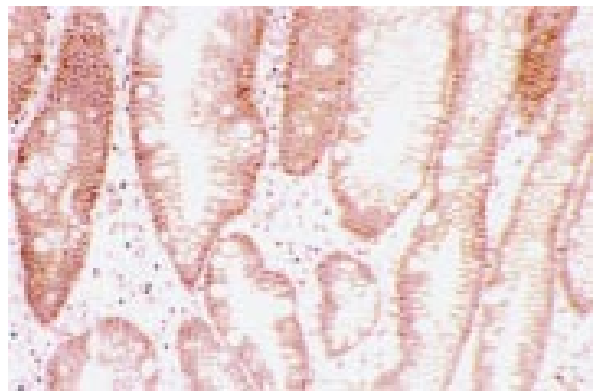

Figure 1 Normal expression of E-cadherin at the intercellular junctions in gastric mucosa, detected by immunohistochemistry. The epithelium shows a degree of intestinal metaplasia.

stratified squamous epithelia, whereas $\mathrm{N}$-cadherin is expressed predominantly in neural tissue. Their location on the cell membrane is not random, but rather they are located in intercellular junctions of the zonula adherens type (fig 1). A further property of the cadherins is that of homotypic binding-"like binding like". Thus, an E-cadherin in one adherent junction will bind to another E-cadherin in the opposing junction of an adjacent epithelial cell, but not to a cell bearing only $\mathrm{P}$-cadherin in the same manner.

Interactions also occur on the cytoplasmic side of the molecule, and a family of cadherin binding proteins has been discovered called catenins. Three of these are currently known: $\alpha$-catenin (102 kDa), $\beta$-catenin $(94 \mathrm{kDa})$, and $\gamma$-catenin $(82 \mathrm{kDa})$. These molecules link cadherins to the actin cytoskeleton and are thought to be essential for normal cadherin function, with $\beta$-catenin or $\gamma$-catenin binding the cadherin molecule and $\alpha$-catenin linking the resulting complex to actin. A further catenin-like molecule, pl20ctn, has also been found bound to E-cadherin, although it does not seem to link cadherins to the actin cytoskeleton. All components of the cadherin-catenin complex are needed for effective cell-cell adhesion.

In addition to their role as mediators of adhesion, cadherins have been shown to be involved in embryonic development, cellular differentiation, and regulation of cellular migration in response to injury. Therefore, the disruption of cadherin function in malignancy is likely to have profound effects on cell morphology and behaviour.

\section{THE ROLE OF E-CADHERIN IN MALIGNANCY}

The disruption of E-cadherin function has been documented in many human solid tumours. Downregulation of E-cadherin has been observed in many epithelial cancers and specific gene mutations have been detected. Particularly interesting is the association between E-cadherin dysfunction and the invasive/ infiltrative growth pattern of neoplastic cells. In poorly cohesive, infiltrative tumours, such as lobular carcinoma of the breast and diffuse gastric carcinoma, ${ }^{3}$ E-cadherin dysfunction and/or mutation is seen. Work on lobular breast cancer ${ }^{4}$ has shown a loss of membranous expression of E-cadherin in these tumours, which was associated with mutations in the cadherin gene in most cases. This loss of expression of E-cadherin on cell membranes, often with relocation to the cytoplasm, has been observed in many malignancies and is an indicator of abnormal cadherin function. Similarly, studies in gastric carcinoma have shown that in tumours of mixed intestinal and diffuse morphology only the diffuse component of the tumour was found to have mutations in the E-cadherin gene. ${ }^{5}$ Further evidence implicating E-cadherin in these particular tumours has come from work identifying a germline mutation in the E-cadherin gene in one particular family with a strong history of gastric carcinoma. This work identified one individual suffering from metachronous diffuse gastric carcinoma and lobular breast cancer, both tumours showing abnormal E-cadherin immunoreactivity. ${ }^{6}$ Conversely, cadherin expression can be seen in tumours that are not of epithelial origin but are epithelioid in phenotype-for example, rare epithelioid Schwann cell tumours of peripheral nerve express E-cadherin, whereas their more common spindle cell counterparts do not. ${ }^{7}$

As well as having "anti-invasive" properties, E-cadherin dysfunction in particular has been shown to affect the differentiation state of a tumour. Using gastric carcinoma again as an example, abnormal E-cadherin expression has been shown to correlate significantly with a poorly differentiated state. ${ }^{8}$ Molecular studies have also indicated that by downstream signalling E-cadherin may also regulate cell proliferation and that dysfunction will increase cell division as well as altering differentiation status. ${ }^{9}$

In view of these observations, and the fact that abnormal cadherin function is seen in many carcinomas (including colorectal carcinoma, prostate carcinoma, oesophageal carcinoma, and bladder carcinoma), we can conclude that the acquisition of dysfunctional cadherin is an important step in tumorigenesis. This is further confirmed when preinvasive neoplastic lesions are studied. In situ lesions of the cervix, ${ }^{10}$ colon, ${ }^{10}$ and oesophagus ${ }^{11}$ have been shown to have abnormal immunostaining for cadherins. In the case of cervical neoplasia, cytoplasmic localisation of E-cadherin was seen in cervical intraepithelial neoplasia (CIN), and was found to correlate positively with CIN grading, ${ }^{12}$ again giving insight into the function of the E-cadherin molecule via morphological assessment.

Techniques such as immunostaining have yielded not only functional data regarding E-cadherin and catenins but have been able to relate abnormal function to clinical parameters. In colorectal carcinoma, for instance, immunohistochemistry and in situ hybridisation have shown that lack of E-cadherin expression correlates with poor tumour differentiation, advanced Dukes's stage, and metastatic potential. ${ }^{13}$ Likewise, a study in breast carcinoma correlated reduced E-cadherin expression (determined by immunohistochemistry) with high histological grade, and shortened disease free survival times. ${ }^{14}$ In transitional cell carcinoma of the bladder, loss of membranous E-cadherin correlates with high histological grade, advanced stage, and reduced survival. ${ }^{15}$ Similar results are seen in gastric ${ }^{16}$ and prostatic ${ }^{17}$ carcinomas. These results show an interesting and consistent trend; however, there is work to be done before such analysis can become a routine method of assessing prognosis.

\section{CATENIN ABNORMALITIES AND MALIGNANCY}

Catenins play an important role in the regulation of cadherin function. Of particular interest is the role of the adenomatous polyposis coli (APC) gene product in the regulation of $\beta$-catenin function. The APC gene was first identified as a putative tumour suppressor gene on chromosome $5 \mathrm{q}$ that was lost in the early stages of colorectal carcinogenesis. The $300 \mathrm{kDa}$ APC protein has been shown to interact with $\beta$-catenin in a multiprotein complex, targeting it for degradation by cellular proteolytic mechanisms. Indeed, APC will compete with E-cadherin to bind the available pool of $\beta$-catenin molecules. In colonic neoplasia, mutated APC molecules are seen which although truncated can still bind $\beta$-catenin. However, these mutant APC proteins do not induce $\beta$-catenin degradation and instead $\beta$-catenin is seen to translocate to the nucleus (fig 2 ), where it interacts with various transcription factors to induce proliferation. In this manner, APC will also sequester free $\beta$-catenin, which cannot then bind to E-cadherin, resulting in loss of cadherin function and proliferation. The epidermal growth factor receptor (EGFR) produces a similar effect, in that phosphorylation of $\beta$-catenin by EGFR will cause destabilisation of the cadherin-catenin complex and disrupted intercellular adhesion. Thus it can be 


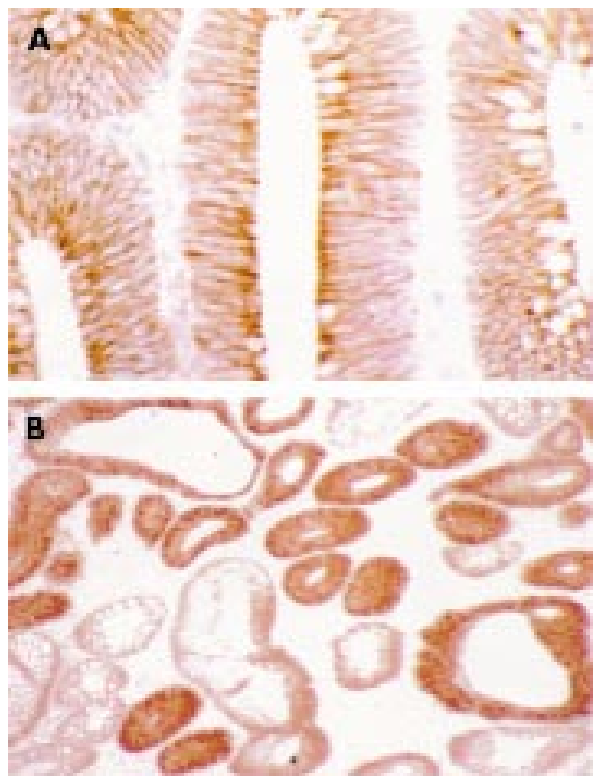

Figure 2 (A) Immunohistochemical staining for $\beta$-catenin in a colonic adenoma. This specimen shows normal membranous localisation of the $\beta$-catenin molecule. (B) Immunostaining for $\beta$-catenin in another colonic adenoma. This section shows abnormal nuclear localisation of the adenomatous polyposis coli (APC)-catenin protein, a change that has been noted in many neoplastic processes.

seen that cadherin function can be disrupted not only by mutation of cadherin itself but by genetic or epigenetic alterations in catenin molecules or their regulators. The results of these complex molecular events can be visualised using the techniques mentioned above. For example, nuclear accumulation of $\beta$-catenin has been observed in hepatocelluar carcinoma $^{18}$ correlating with mutation in the $\beta$-catenin gene. In a similar manner nuclear $\beta$-catenin and non-membranous E-cadherin patterns are seen in Barrett's oesophagus ${ }^{19}$ and in colorectal adenomas. ${ }^{10}$ Mutation of $\beta$-catenin has also been seen in Wilms's tumour ${ }^{20}$ and prostate carcinoma. ${ }^{21}$

\section{CONCLUSION}

Studies on the expression and subcellular localisation of the E-cadherin-catenin complex have shown that morphological analysis can give valuable information regarding the function of an important molecule in cancer development, and it is likely that in the future more molecules will prove to be amenable to a similar approach. Already new technologies make the link between morphological assessment and molecular analysis even more intimate. For example, recent developments in laser assisted microdissection mean that this procedure can now be used to capture single cells from routinely processed slides, which can subsequently undergo various molecular analyses. In fact, this technique has already been used in the analysis of E-cadherin mutations. ${ }^{22}$

Novel molecular techniques will play a bigger role and the detection of mutations will undoubtedly become more and more relevant in the clinical setting. However, it is conceivable that morphology and genetics, phenotype and genotype will continue to have the same intimate relation that they have enjoyed since the days of Mendel.

\section{Authors' affiliations}

R J Campbell, M Pignatelli, Division of Histopathology, Department of Pathology and Microbiology, University of Bristol, Bristol Royal Infirmary, Marlborough Street, Bristol BS2 8HW, UK

\section{REFERENCES}

1 Edelman GM, Crossin KL. Cell adhesion molecules: implications for a molecular histology. Annu Rev Biochem 1991;60:155-90.

2 Smith MEF, Pignatelli M. The molecular histology of neoplasia: the role of the cadherin/catenin complex. Histopathology 1997;31:107-11.

3 Becker KF, Atkinson M, Reich U, et al. E-Cadherin gene mutations provide clues to diffuse type gastric carcinomas. Cancer Res 1994:54:3845-52.

4 De Leeuw WJ, Berx G, Vos CB, et al. Simultaneous loss of E-cadherin and catenins in invasive lobular breast cancer and lobular carcinoma in-situ. J Pathol 1997; 183:404-1 1

5 Machado JC, Soares P, Carneiro F, et al. E-cadherin gene mutations provide a genetic basis for the phenotypic divergence of mixed gastric carcinomas. Lab Invest 1999:79:459-65.

6 Keller G, Vogelsang H, Becker I, et al. Diffuse type gastric and lobular breast carcinoma in a familial gastric cancer patient with an E-cadherin germline mutation. Am J Pathol 1999;155:337-42.

7 Smith MEF, Cowely GP, Dogan A, et al. E-cadherin is a differentiation antigen of normal Schwann cells and is expressed in epithelioid Schwann cell tumors. J Pathol 1994;173:181A.

8 Karayiannakis AJ, Syrigos KN, Chatzigianni E, et al. E-cadherin expression as a differentiation marker in gastric cancer Hepatogastroenterology 1998:45:2437-42.

9 Jawhari AU, Farthing MG, Pignatelli M. The E-cadherin/epidermal growth factor receptor interaction: a hypothesis of reciprocal and reversible control of intercellular adhesion and cell proliferation. J Pathol 1999; 187: 155-7

10 Valizadeh A, Karayiankis AJ, el-Hariry l, et al. Expression of E-cadherin-associated molecules (alpha-, beta-, and gamma-catenins and p120) in colorectal polyps. Am J Pathol 1997:150:1977-84.

11 Bailey T, Biddlestone L, Shepherd N, et al. Altered cadherin and catenin complexes in the Barret's esophagus-dysplasia-adenocarcinoma sequence: correlation with disease progression and dedifferentiation. Am J Pathol 1998;152:135-44.

12 Vessey CJ, Wilding J, Folarin N et al. Altered expression and function of E-cadherin in cervical intraepithelial neoplasia and invasive squamous cell carcinoma. J Pathol 1995:176:151-9

13 Dorudi S, Sheffield JP, Poulsom R, et al. E-cadherin expression in colorectal cancer. An immunocytochemical and in situ hybridisation study. Am J Pathol 1993;142:981-6.

14 Siitonen SM, Kononen JT, Helin HJ, et al. Reduced E-cadherin expression is associated with invasiveness and unfavourable prognosis in breast cancer. Am J Clin Pathol 1996; 105:394-402

15 Syrigos KN, Krausz T, Waxman J, et al. E-cadherin expression in bladder cancer using formalin fixed paraffin imbedded tissues: correlation with histopathological grade, tumor stage and survival. Int J Cancer 1995;64:367-70.

16 Jawhari A, Jordan S, Poole S, et al. Abnormal immunoreactivity of the E-cadherin-catenin complex in gastric carcinoma: relationship with patient survival. Gastroenterology 1997;1 12:46-54.

17 Richmond PJ, Karayiannakis AJ, Nagafuchi A, et al. Aberrant E-cadherin and alpha-catenin expression in prostate cancer: correlation with patient survival. Cancer Res 1997:57:3189-93.

18 Nhieu JT, Renard CA, Wei Y, et al. Nuclear accumulation of mutated beta-catenin in hepatocellular carcinoma is associated with increased cell proliferation. Am J Pathol 1999;155:703-10.

19 Jankowski JA, Newham PM, Kandemir O, et al. Differential expression of E-cadherin in normal, metaplastic and, dysplastic oesophageal mucosa: a putative biomarker. Int J Oncol 1994;4:441-8.

20 Koesters R, Ridder R, Kopp-Scneider A, et al. Mutational activation of the beta-catenin proto-oncogene is a common event in the developmen of Wilm's tumors. Cancer Res 1999;59:38880-2.

21 Voeller HJ, Trucia Cl, Gelmann EP. Beta-catenin mutations in human prostate cancer. Cancer Res 1998:58:2520-3.

22 Becker I, Becker KF, Rohrl MH, et al. Laser-assisted preparation of single cells from stained histological slides for gene analysis. Histochem Cell Biol 1997; 108:447-51 